\title{
Editorial
}

\section{Minnesota coding and the prevalence of ECG abnormalities}

The electrocardiogram (ECG) has been used in population studies for over 40 years. The value of such research from an electrocardiographic standpoint relates to determining the prognostic value of various ECG abnormalities. The prevalence and prognostic significance of ECG abnormalities have recently been comprehensively reviewed, ${ }^{1}$ following an earlier extensive summary of the ECG in epidemiology and clinical trials. ${ }^{2}$ The most recent survey in the current issue of Heart from a Belgian team ${ }^{3}$ concentrates on the prevalence of various ECG abnormalities in the general population through an analysis of ECGs from 47358 men and women; as such, it builds on earlier reports from the same authors where the prognostic significance of ECG abnormalities in a subset of the same population was reported. ${ }^{45}$

\section{Methods and accuracy of coding}

Most population based studies make use of the Minnesota Code, first introduced in $1960^{6}$ and subsequently extended to incorporate serial comparison in $1983 .{ }^{7}$ An updated manual is due for publication shortly. Coding of an ECG can be done manually as in the paper of De Bacquer and colleagues $^{3}$ or it can be done by automated methods. ${ }^{8}$ Both approaches, however, are subject to error ${ }^{9} 10$ and $100 \%$ reliance cannot be placed on either the coding of one individual or an automated technique. In the case of manual coding, it is better to have two individuals coding independently and then reaching a consensus in the event of disagreement, or if the coding centre is large enough, a third (more experienced) observer can adjudicate. Automated codings should be reviewed by an observer who effectively provides a second opinion. Even then, a second observer is also of value when there is disagreement between an automated report and the first observer. There is much that can be regarded as subjective in coding an ECG when $1 \mu \mathrm{V}$ in $\mathrm{R}$ amplitude can significantly change a classification from normal to highly abnormal, or vice versa-for example, in the lateral leads, where one of the criteria for abnormality is $\mathrm{Q} \geqslant 40 \mathrm{~ms}$ and $\mathrm{R} \geqslant 300 \mu \mathrm{V}$ in aVL (1-1-3 lateral - vide infra).

One early report comparing six approaches to coding ${ }^{9}$ found $84.5 \%, 85 \%$, and $87.5 \%$ accuracy in coding 522 ECGs using three different manual approaches where the gold standard reflected consensus among the six approaches. Three automated techniques had accuracies of $55.2 \%, 77.3 \%$, and $77.9 \%$ in the same test. With more recently developed automated methods, ${ }^{10}$ repeat codings showed discrepancies in 16\% for ECGs recorded 15-30 minutes apart using marked electrode positions and in $19 \%$ for ECGs recorded 2-3 weeks apart.

\section{Minnesota Code}

The Minnesota Code ${ }^{6}$ itself does not produce an interpretation of an ECG but, rather, provides a classification of electrocardiographic morphology on the basis of rigid criteria-for example, Q duration in lead II $\geqslant 40 \mathrm{~ms}$ constitutes a code 1-1-2 (posterior), where a $Q$ wave is defined as a negative wave that is followed by a positive (R) wave of defined minimum amplitude and duration. Thus, for example, a QS complex of any duration in lead II does not constitute a code 1-1-2. Codes 1-that is, codes beginning with 1 -deal with $Q$ waves of varying duration and amplitude in a hierarchical way with codes $1-1-\mathrm{x}$ (where $\mathrm{x}=1$, 2 ...7) generally representing $Q$ waves of larger magnitude, and hence clinical significance, than codes 1-2-y (where $y=1,2 \ldots 8$ ) which in turn are more significant than codes $1-3-z$ (where $z=1,2 \ldots 6$ ). Table 1 broadly summarises the codes. As another example, a clearly inverted $T$ wave in V3 might be classified as 5-2 (anterior) in the presence of a QRS complex with normal duration. The exceptions to the application of criteria for pattern recognition are the Wolff-Parkinson-White (WPW) pattern and arrhythmias, which require to be interpreted by a reviewer who then assigns the relevant code-for example, atrial fibrillation is coded as 8-3-1.

While codes 1 are often mapped to a diagnosis of myocardial infarction, with, for example, 1-1-1 being regarded as diagnostic and $1-3-4$ as possible myocardial infarction, the Minnesota Code per se does not draw such conclusions. Similarly, while 5-2 might be interpreted as a pattern that provides evidence of ischaemic heart disease in population studies such as that in this issue, ${ }^{3}$ some cardiologists might argue that this is an unreliable approach to adopt, given the many causes of $\mathrm{T}$ wave change.

The code itself has several deficiencies. For example, the criteria for high voltage are neither age nor sex dependent and it is well known that such criteria indeed should be so, given the current state of knowledge of the normal limits of the 12 lead ECG.${ }^{11}$ As another example, the code does not classify low $\mathrm{R}$ waves in the precordial leads apart from reversed $R$ wave progression.

It would be fair to say that most cardiologists are not conversant with the Minnesota Code and, indeed, need not be in terms of daily clinical routine. The code itself has remained essentially unchanged through the years and only in recent times has there been any attempt to produce an alternative approach, as was done by Rautaharju (one of the authors of the original Minnesota Code) and colleagues. ${ }^{12}$ The benefit of Minnesota coding lies in its reputed ability to afford an objective comparison of findings in various populations independent of the more subjective views of individual cardiologists who might be called upon to report an ECG. Furthermore, in clinical trials such as the West of Scotland Coronary Prevention Study (WOSCOPS), ${ }^{13}$ Minnesota coding proved invaluable in providing criteria on which to base electrocardiographic aspects of end points. ${ }^{14}$

Table 1 Classifications used by the Minnesota Code

\begin{tabular}{ll}
\hline Minnesota Code & ECG abnormality \\
\hline $1-1-1 \ldots \ldots .1-3-6$ & Q waves \\
$2-1 \ldots \ldots .5$ & QRS axis deviation \\
$3-1 \ldots \ldots .3$ & High amplitude R waves \\
$4-1-1 \ldots \ldots .4-4$ & ST junction $(J)$ and segment depression \\
$5-1 \ldots \ldots .4$ & T wave items \\
$6-1 \ldots \ldots 6-8$ & A-V conduction defect \\
$7-1-1 \ldots \ldots .8$ & Ventricular conduction defect \\
$8-1-1 \ldots \ldots .8-9$ & Arrhythmias \\
$9-1 \ldots \ldots 9-8-2$ & Miscellaneous including ST segment elevation (9-2)
\end{tabular}

Codes $1,4,5$, and 9-2 (ST elevation) are grouped by leads, resulting in three subclassifications of anterolateral, posterior (inferior) and anterior. 


\section{Belgian study}

With the foregoing in mind, it is of interest to review the Belgian report by De Bacquer and colleagues. ${ }^{3}$ The ECGs of 47358 men and women participating in four Belgian epidemiological studies from the mid 1970s onwards have been combined in a form of meta analysis. Approximately $55 \%$ of the ECGs were coded by one individual while, for the remainder, a second reviewer assisted. The authors classified the ECGs according to various Minnesota Code categories - for example, major ECG abnormality was defined as pathological Q waves (codes 1-1 and 1-2), marked ST depression (codes 4-1 and 4-2) and/or T wave inversion (codes 5-1 and 5-2), bundle branch block (codes 7-1 and 7-2) or some significant arrhythmias. Minor ECG abnormalities were defined as high voltage, axis deviation and lesser degrees of ST-T wave abnormality $(4-3,5-3)$ according to the Minnesota Code. A different form of classification of "ischaemic ECG" was defined as pathological Q waves (any code 1), ST and/or T wave inversion of any degree (any code 4 or 5) or left bundle branch block (code 7-1-1). Left ventricular hypertrophy was defined as a combination of high voltage and either ST depression or T wave inversion, again on the basis of appropriate Minnesota Codes.

In addition to electrocardiographic classification, a clinical classification was available for each patient. In particular, prevalent coronary heart disease was defined as angina pectoris or a positive history of acute myocardial infarction (self reported) or Q wave evidence (codes 1-1 and 1-2) of an old myocardial infarction on the resting ECG. Lifestyle characteristics were also recorded-for example, smoking habits, living alone, etc, while clinical data on hypertension, high total cholesterol ( $>6.5 \mathrm{mmol} / \mathrm{l}$ or $>250 \mathrm{mg} / \mathrm{dl}$ ), and Rose questionnaire ${ }^{15}$ based angina pectoris were also noted.

\section{Sex differences}

Of particular interest is the fact that women have significantly more ST changes $(2.6 \% v 2.3 \%)$ and $\mathrm{T}$ wave abnormalities $(7.6 \%$ v $6.5 \%)$ on their resting ECG compared to men when adjustment is made for age, although the absolute differences in percentage terms were small $(0.3 \%$ and $1.1 \%$, respectively). This sex difference persisted in virtually all age deciles from 25 to 74 years. In a further analysis adjusted for age and other prognostic factors, only the ST changes remained significantly more common in women. Notwithstanding, the prevalence of angina is similarly higher in females than in males $(6.0 \% v$ $5.0 \%$ ) in all but the highest age group (65-74 years). In the same vein, the prevalence of coronary heart disease was found to be higher in women than men up to age 55 with a reversal of the situation above that age. On the contrary, the prevalence of a history of myocardial infarction was higher in males than in females $(3.6 \% v 1.5 \%)$ with the exception of the youngest age range (25-34 years) where there was an increased prevalence in women $(0.1 \% v 0 \%)$.

Although it is difficult to reconcile all these data, somewhat similar results were found in the Whitehall II study. ${ }^{16}$ In that study of 6895 male and 3413 female civil servants aged between 35 and 55 years on entry, men had a higher prevalence of "diagnostic" $\mathrm{Q}$ waves, equivocal $\mathrm{Q}$ waves, and pain suggestive of myocardial infarction than women; $4.0 \%$ of women and $2.4 \%$ of men had self reported Rose questionnaire angina, while both men and women with angina had a higher prevalence of ST-T abnormalities compared to those without. The authors concluded that the "relationship between Rose angina and ischaemia (albeit imprecisely measured) appears weaker in men than in women".

In addition, 10 year follow up data ${ }^{4}$ from a subset of the Belgian cohort support the hypothesis that women with ischaemic ECG changes defined as any of Minnesota Codes $1-3,4-1$ to $4-3,5-1$ to $5-3$ or $7-1-1$, have the same increased risk of death due to cardiovascular disease as men, with the multivariately adjusted risk ratio being 2.45 (95\% confidence interval (CI) 1.70 to 3.53 ) for men and 2.16 (95\% CI 1.30 to 3.58 ) for women.

Association of ECG abnormalities with lifestyle and coronary heart disease

In the study virtually all ECG abnormalities were linked with coronary heart disease. ${ }^{3}$ Even those individuals with only minor ECG changes had a multivariately adjusted risk ratio of 1.28 (95\% CI 1.13 to 1.44 ) of having coronary heart disease. This ties in with minor ECG ST-T changes (codes 4-2, 4-3, 5-2, 5-3) in WOSCOPS middle aged males having significant prognostic value in the prediction of fatal or non-fatal myocardial infarction both on a univariate and multivariate ${ }^{17}$ basis.

One of the strengths of the Belgian study is the ability to relate the prevalence of ECG abnormalities with the lifestyle characteristics. Ischaemic-like ECG changes were linked by the authors to obesity, diabetes, region, and employment status as well as age and overt coronary heart disease. The authors suggest that these are new findings - for example, being employed was linked with a lower prevalence of ischaemic ECG findings. Smoking had no effect on the prevalences of ECG abnormalities.

It would have been of interest to know what the prevalence of silent myocardial infarction might have been, given that the lack of a history of acute myocardial infarction might have been related to the prevalence of abnormal Q waves on the resting ECG. The authors' results suggest that the prevalence of the relevant Minnesota Code abnormalities $(1-1,1-2)$ is in the order of $50 \%$ of the prevalence of acute myocardial infarction by history, except in one particular study - the Belgian jobstress project which studied the independent relation of perceived job stress with the incidence of fatal or non-fatal myocardial infarction. This study, which was the most recent, showed that there were $0.5 \%$ of ECGs with $Q$ wave evidence suggestive of myocardial infarction in the absence of any relevant history both in men and women. On the other hand, using the authors' expanded definition of Q/QS abnormalities involving all codes 1 , namely 1-1 to $1-3$, there was a higher prevalence of $\mathrm{Q} / \mathrm{QS}$ codes in women compared to a history of acute myocardial infarction $(2.3 \% v 1.5 \%)$ and similarly in men up to 54 years, but a reversal in men thereafter, giving an overall higher prevalence of acute myocardial infarction than $\mathrm{Q}$ waves $(3.6 \% v 3.2 \%)$ in men. These data make it difficult to draw any firm conclusions on the prevalence of silent myocardial infarction.

\section{Miscellaneous}

The prevalence of left ventricular hypertrophy was found to be significantly higher in males than females $(0.7 \% v$ $0.5 \%)$. It is suggested that inadequate criteria may in part be responsible for this finding, because criteria thresholds should be lower in women than men, but this is not the case in the Minnesota Code. The same cannot be said of right bundle branch block, the incidence of which was almost three times as high in males as in females, in all age ranges.

The prevalence of arrhythmias is also of interest. The most commonly studied arrhythmia, namely atrial fibrillation, was found to increase in prevalence as age increased, as is well known. ${ }^{18}$ However, although the Belgian authors indicate that the overall prevalence of $0.55 \%$ in men and $0.33 \%$ in women is comparable with other studies, a prevalence of $2.7 \%$ in males and $1.53 \%$ in females in the 65-74 years age range is a little lower than found by others - for example, Feinberg and colleagues found a prevalence of $5.9 \%$ in the over $65 \mathrm{~s}$, with rates of $3 \%$ in the $65-69$ age group, and $5 \%$ in the $70-74$ age group. ${ }^{18}$ 


\section{Conclusions}

The authors themselves acknowledge that variation in different studies regarding the prevalence of ECG findings can be attributed in the main to observer bias in keeping with what has been outlined above. In this Belgian study, it should also be noted that $55 \%$ of ECGs were reviewed by a single individual. Furthermore, population sampling is clearly open to variation, and in this meta-analysis ${ }^{3}$ no-one was excluded on the basis of clinical history while there was varying participation in the four separate studies. Notwithstanding, while absolute prevalences might be open to discussion, the relative prevalences according to age in particular are clear. Virtually every ECG abnormality, with the exception of high $\mathrm{R}$ waves and WPW pattern, increases in prevalence with increasing age.

This Belgian study shows that there is still much to be gleaned from careful analysis of the ECG in large populations. It is generally recognised that the usefulness of a screening test for a particular abnormality depends among other things on the prevalence of the abnormality, and to this end, the Belgian data derived from 47358 subjects, including 12637 women, add significantly to existing knowledge of the prevalence of ECG abnormalities in a general population.

PETER W MACFARLANE

University of Glasgow, Department of Medical Cardiology,

Royal Infirmary, Glasgow G31 2ER, UK

peter.w.macfarlane@clinmed.gla.ac.uk

1 Ashley EA, Raxwal VK, Froelicher VF. The prevalence and prognostic significance of electrocardiographic abnormalities. Current Problems in Cardiology 2000;25:7-72.

2 Rautaharju PM. Electrocardiography in epidemiology and clinical trials. In: Macfarlane PW, Lawrie TDV, eds. Comprehensive electrocardiology, vol 2. Oxford: Pergamon Press, 1989, 1219-66.
3 De Bacquer D, De Backer G, Kornitzer M, et al. Prognostic value of ECG findings for total, cardiovascular disease, and coronary heart disease death in men and women. Heart 1998;80:570-7.

4 De Bacquer D, De Backer G, Kornitzer M, et al. Prognostic value of ischemic electrocardiographic findings for cardiovascular mortality in men and women. f Am Coll Cardiol 1998;32:680-5.

5 De Bacquer D, De Backer G, Kornitzer M. Prevalences of ECG findings in large population based samples of men and women. Heart 2000;84:625-33.

6 Blackburn H, Keys A, Simonsen E, et al. The electrocardiogram in population studies. A classification system. Circulation 1960;21:1160-75.

7 Prineas RJ, Crowe RS, Blackburn H. The Minnesota Code manual of electrocardiographic findings. Bristol: John Wright, 1982.

8 Macfarlane PW, Latif S. Automated Serial ECG comparison based on the Minnesota Code. F Electrocardiol 1996;29(suppl):29-34.

9 Tuinstra CL, Rautaharju PM, Prineas RJ, et al. Comparison of the operational value of six systems to assess the Minnesota Code. In: de Padua F, Macfarlane PW, eds. New frontiers of electrocardiology. Chichester: Wiley, 1981,487-92.

10 de Bruyne MC, Kors JA, Visentin S, et al. Reproducibility of computerized ECG measurements and coding in a non-hospitalized elderly population. $\mathcal{F}$ Electrocardiol 1998;31:189-95.

11 Macfarlane PW, Lawrie TDV, eds. Comprehensive electrocardiology, vol 3. Oxford: Pergamon Press, 1989:1441-1526.

12 Rautaharju PM, Park LP, Chaitman BR, et al. The Novacode criteria for classification of ECG abnormalities and their clinically significant progression and regression. F Electrocardiol 1994;31:157-87.

13 Shepherd J, Cobbe SM, Ford I, et al. Prevention of coronary heart disease with pravastatin in men with hypercholesterolemia. N Engl f Med 1995; 33:1301-7.

14 West of Scotland Coronary Prevention Study Group. A Coronary primary prevention study of scottish men aged 45-64 years: trial design. $\mathcal{f}$ Clin Epidemiol 1992;45:849-60.

15 Rose G. Self administration of a questionnaire on chest pain and intermittent claudication. Br F Prev Soc Med 1977;31:42-53.

16 Nicholson A. White I, Macfarlane P, et al. Rose questionnaire angina in younger men and women: gender differences in the relationship to cardiovascular risk factors and other reported symptoms. F Clin Epidemiol 1999; 52:337-46.

17 West of Scotland Coronary Prevention Group. Baseline risk factors and their association with outcome in the West of Scotland coronary prevention study. Am f Cardiol 1997;79:756-72.

18 Feinberg WM, Blackshear JL, Laupacis A, et al. Prevalence, age distribution, and gender of patients with atrial fibrillation. Arch Intern Med 1995;155: 469-73.

\section{IMAGES IN CARDIOLOGY}

\section{Seven enigmatic dwarfs}

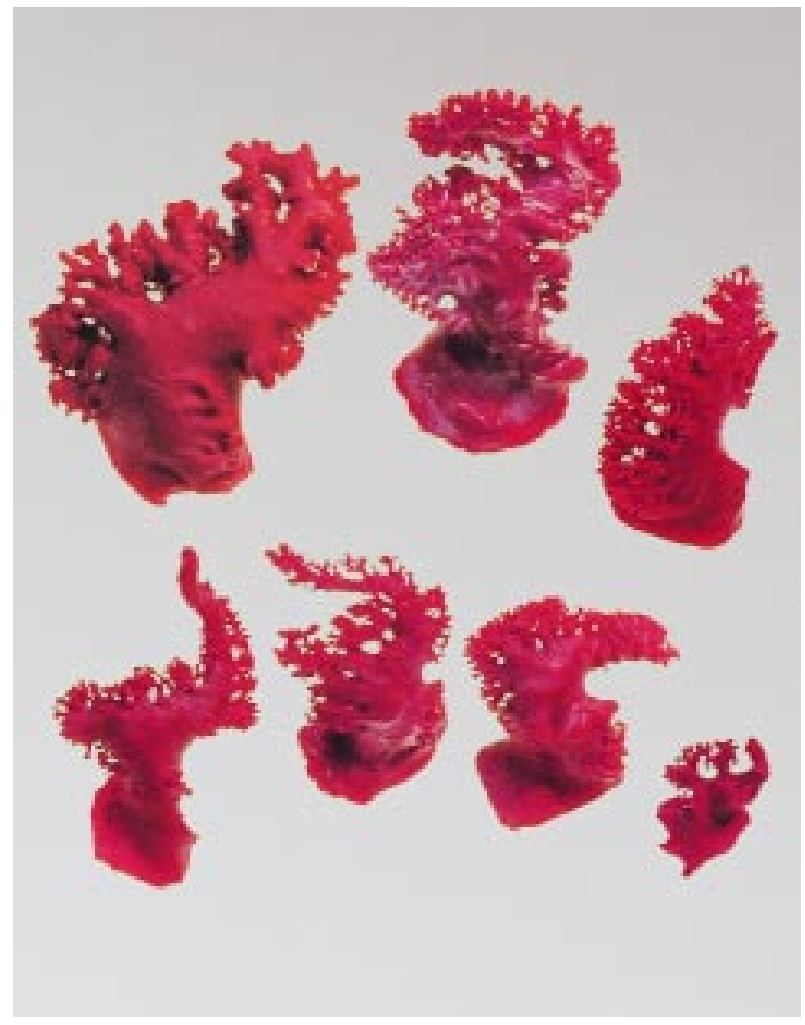

Casts of the left atrial appendage, obtained at postmortem examination, show its variable and complex morphology. One left atrial appendage contained a thrombus (first from the left, upper row). Ante mortem, two patients had atrial fibrillation (first and second from the left, upper row); the remaining five patients had sinus rhythm. Haemodynamic changes in atrial fibrillation may lead to enlargement of the left atrial appendage. A patent foramen ovale was found in one patient (first from the right, lower row). The smaller volume of the left atrial appendage might be a consequence of a left-to-right shunt.

CLAUDIA STÖLLBERGER GÜNTHER ERNST JOSEF FINSTERER 